\title{
DETERMINAÇÃO DO COEFICIENTE DE ATIVIDADE EM DILUIÇÃO INFINITA DE HIDROCARBONETOS EM FURFURAL A 298,15 K POR SPME-GC/FID
}

\author{
Filipe Arantes Furtado e Gerson Luiz Vieira Coelho* \\ Departamento de Engenharia Química, Instituto de Tecnologia, Universidade Federal Rural do Rio de Janeiro, Rod. BR 465, \\ km 7, 23890-000 Seropédica - RJ, Brasil
}

Recebido em 8/2/10; aceito em 25/6/10; publicado na web em 22/9/10

\begin{abstract}
DETERMINATION OF ACTIVITY COEFFICIENT AT INFINITE DILUTION OF HYDROCARBONS IN FURFURAL AT 298.15 K THROUGH SPME-GC/FID. In this work a new method (SPME-GC/FID) was developed to analyze the activity of binary liquid mixtures. The purpose is to demonstrate that SPME is capable to be used to determinate activity coefficients at infinite dilution knowing the fiber properties, with a lower cost than the conventional methods encountered in literature such as GLC. The activity coefficients at infinite dilution in furfural for $n$-hexane, $n$-heptane and cyclohexane at $298.15 \mathrm{~K}$ was determined using SPME and deviations of literature data was about $7 \%$.
\end{abstract}

Keywords: partition coefficients; activity coefficients; gas chromatography.

\section{INTRODUÇÃO}

Um soluto está infinitamente diluído quando as moléculas deste soluto estão completamente envolvidas por moléculas de solvente; neste caso, a fração molar do soluto em solução tende a zero e a fração molar do solvente tende a um. Nesta conformação a não idealidade deste soluto em solução é máxima, sendo representada pelo coeficiente de atividade na diluição infinita $\left(\gamma^{\infty}\right)$.

Tais coeficientes são de grande importância, visto que uma vez determinados é possível predizer o equilíbrio entre dois componentes determinando-se os parâmetros de modelos matemáticos que descrevem as variações das energias de excessos e atividade dos componentes dessas misturas. Com isso, equipamentos que têm como fundamentação básica o equilíbrio entre fases podem ser projetados com maior precisão, obtendo-se compostos mais puros. Os coeficientes de atividade em diluição infinita podem ainda ser utilizados para a seleção de solventes capazes de remover de uma solução um dado componente em maior quantidade que outros, definindo um parâmetro denominado seletividade, que consiste em uma relação entre os coeficientes de atividade em diluição infinita de dois solutos em um mesmo solvente a uma mesma temperatura. ${ }^{1}$

Um destes fatores pode ser observado na indústria do petróleo, em que fenol ou furfural são empregados na remoção de compostos aromáticos polinucleados de óleos lubrificantes num processo denominado desaromatização. A presença destes compostos aromáticos prejudica a viscosidade do óleo produzido, quando este é submetido a variações de temperatura, ${ }^{2}$ ou seja, este é um processo que necessita dos parâmetros acima citados para a seleção do solvente adequado.

Muitas técnicas vêm sendo desenvolvidas ao longo dos anos para a determinação dos coeficientes de atividade em diluição infinita para misturas líquidas, sendo algumas mais complexas e custosas, como o GLC (Gas-Liquid Chromatography), ${ }^{3-5}$ e absorção gasosa com gás inerte não solúvel (Gas-Stripping) ${ }^{5,6} \mathrm{Na}$ técnica de GLC uma coluna cromatográfica com um suporte inerte é empacotada com um solvente líquido não volátil como fase estacionária. Em seguida, volumes reduzidos de soluto são injetados, de modo a se determinar o tempo de retenção do soluto no solvente utilizado como fase estacionária. O coeficiente de atividade na diluição infinita é determinado a partir

*e-mail: coelho@ufrrj.br da aferição do tempo de retenção do soluto e de outras propriedades como temperatura e queda de pressão ao longo da coluna, fluxo de gás de arraste e massa de soluto na coluna. No caso do gas-stripping uma solução diluída de um sistema binário é alimentada em uma célula de equilíbrio com temperatura controlada. A partir desse ponto um gás inerte é borbulhado com fluxo constante a partir da base da célula e os componentes de interesse migram da fase líquida para a fase vapor. Atingindo o equilíbrio, medições constantes da composição na fase líquida e vapor são realizadas de modo a se determinar a taxa de retirada de um dos componentes da solução e, assim, determinar o coeficiente de atividade na diluição infinita.

Para sistemas poliméricos, os coeficientes de atividade em diluição infinita são determinados a partir dos coeficientes de partição soluto-polímero, sendo estes coeficientes determinados por métodos gravimétricos ou por cromatografia inversa. ${ }^{7} \mathrm{Na}$ cromatografia inversa o material a ser analisado fica no interior de uma coluna cromatográfica, semelhante à fase estacionária polimérica de uma coluna capilar, e utiliza-se o método do índice de retenção linear em temperatura programada (IRLTP; LTPRI = Linear Temperature-Programmed Retention Index), que é análogo ao índice de Kovats. ${ }^{8}$

Estudos mostram as possibilidades e vantagens do uso de uma técnica alternativa, como SPME (Solid Phase Microextraction), para a determinação do coeficiente de partição. ${ }^{9,10}$

SPME é uma microtécnica em que os processos de extração e pré-concentração de analitos ocorrem em dimensões não usuais. Seu dispositivo básico consiste em um fino bastão de fibra óptica de sílica fundida de $100 \mu \mathrm{m}$ de diâmetro, contendo $10 \mathrm{~mm}$ de uma das extremidades recoberta com um polímero adsorvente (a fase estacionária) de diferentes espessuras e materiais como, por exemplo, o polidimetilsiloxano (PDMS), poliacrilato (PA) e o polidimetilsiloxano/divinilbenzeno (PDMS/DVB). ${ }^{11}$ A SPME deve ser utilizada em conjunto com um equipamento analítico, de modo a quantificar o material extraído pelo polímero da fibra. Os equipamentos analíticos mais utilizados são o cromatógrafo a gás (CG; $\mathrm{GC}=$ Gas Chromatograph) ou a cromatografia liquida de alta eficiência (CLAE; HPLC = High Performance Liquid Chromatography). ${ }^{12}$

Neste trabalho foi desenvolvido um novo método de determinação para os coeficientes de atividade em diluição infinita, considerando-se o equilíbrio de três fases distintas em um sistema fechado contendo 
uma fase líquida, formada pela mistura solvente-soluto; uma fase gasosa, formada pela mistura vapor do solvente, vapor do soluto e um gás inerte; e, ainda, a fase polimérica da fibra de SPME. Foram também desenvolvidas equações análogas às já existentes na literatura, que possibilitaram o desenvolvimento do método e permitiram a comparação com métodos existentes e já estabelecidos na literatura para o sistema proposto.

\section{Teoria}

Para a determinação dos coeficientes de atividade em diluição infinita utilizando a SPME, é necessária a definição de um sistema fechado, contendo fase líquida, fase gasosa e fase estacionária polimérica da fibra de SPME, mostrado na Figura 1.

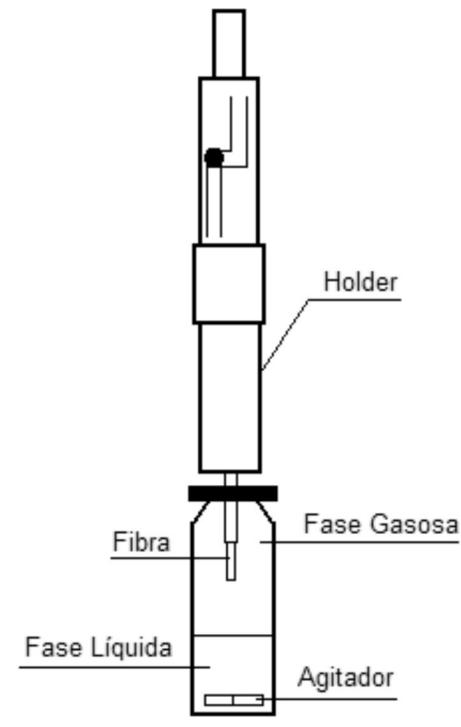

Figura 1. Esquema de um sistema fechado contendo: fase líquida, fase gasosa e a fibra de SPME

Os coeficientes de partição de um dado soluto entre as fases gasosa e polimérica, e entre as fases líquida e gasosa, podem ser determinados pelas Equações 1 e 2 .

$$
\begin{gathered}
K_{f g}=\frac{C_{f}}{C_{g}}=\frac{n_{1}^{f}}{V_{f}} \frac{V_{g}}{n_{1}^{g}} \\
K_{L g}=\frac{C_{L}}{C_{g}}=\frac{n_{1}^{L}}{V_{L}} \frac{V_{g}}{n_{1}^{g}}
\end{gathered}
$$

onde $\mathrm{K}_{\mathrm{fg}}$ é o coeficiente de partição entre o revestimento da fibra e a amostra gasosa, $\mathrm{K}_{\mathrm{Lg}}$ é o coeficiente de partição entre a fase líquida e a fase gasosa, $\mathrm{C}_{\mathrm{L}}, \mathrm{C}_{\mathrm{f}}$ e $\mathrm{C}_{\mathrm{g}}$ são as concentrações do soluto na fase líquida, no revestimento da fibra e na fase vapor, $\mathrm{n}_{1}{ }^{\mathrm{L}}, \mathrm{n}_{1}{ }^{\mathrm{g}}$ e $\mathrm{n}_{1}{ }^{\mathrm{f}}$ são o número de moles de soluto na fase líquida, na fase gasosa e no revestimento da fibra, $V_{f}, V_{g}$ e $V_{L}$ são, respectivamente, os volumes do revestimento da fibra, da fase vapor e da fase líquida.

Quando o equilíbrio deste sistema é atingido, a concentração de soluto na fase gasosa será a mesma nas Equações 1 e 2; substituindose a Equação 2 na Equação 1 é obtida a Equação 3, que relaciona o coeficiente de partição líquido - vapor com o coeficiente de partição fibra - vapor.

$$
K_{L g}=\frac{C_{L}}{C_{f}} K_{f g}
$$

Considerando-se o sistema fechado, o que implica em uma não variação da massa total com o tempo, pode-se realizar o balanço de massa para o soluto, como é mostrado na Equação 4, onde $\mathrm{C}_{\mathrm{Lo}}$ é a concentração inicial de soluto na fase líquida e $\mathrm{n}_{1}$ é o número de moles totais do soluto no sistema.

$$
n_{1}=C_{f} V_{f}+C_{L} V_{L}+C_{g} V_{g}=C_{L 0} V_{L}
$$

Considerando-se constantes os volumes das fases gasosa, líquida e polimérica, visto que no equilíbrio a massa transferida da fase líquida para a fase vapor não altera de forma expressiva o volume do líquido, a Equação 4 pode ser reescrita, obtendo-se a Equação 5.

$$
C_{L}=C_{L 0}-C_{f} \frac{V_{f}}{V_{L}}-C_{g} \frac{V_{g}}{V_{L}}
$$

Substituindo-se a Equação 1 e a Equação 5 na Equação 2, é obtida a Equação 6, que permite a determinação do coeficiente de partição líquido - vapor conhecendo-se as propriedades da fibra, o número de moles inicial de soluto na fase líquida $\left(\mathrm{n}_{0}{ }^{\mathrm{L}}\right)$ e o número de moles absorvidos/adsorvidos pelo revestimento polimérico.

$$
K_{L g}=\left[K_{f g} V_{f}\left(\frac{n_{0}^{L}}{n_{1}^{f}}-1\right)-V_{g}\right] \frac{1}{V_{L}}
$$

Utilizando a Equação 7, uma modificação da equação proposta por Everett e Cruickshank, é possível determinar o coeficiente de atividade em diluição infinita. ${ }^{3,4}$ Onde $\mathrm{n}_{2}{ }^{\mathrm{L}}$ é o número de moles de solvente na fase líquida, $\mathrm{P}_{1}$ sat é a pressão de saturação do soluto, $\mathrm{R}$ a constante dos gases, $\mathrm{T}$ a temperatura, $\mathrm{B}_{11}$ o segundo coeficiente de Virial do soluto e $\mathrm{v}_{1}{ }^{\circ} \mathrm{o}$ volume molar do soluto.

$$
\ln \gamma_{1}^{\infty}=\ln \left(\frac{n_{2}^{L} R T}{K_{L g} P_{1}^{s a t} V_{L}}\right)-\frac{P_{1}^{s a t}\left(B_{11}-v_{1}^{0}\right)}{R T}
$$

Para utilizar a Equação de Everett e Cruickshank em termos da densidade do solvente, basta substituir nesta a Equação 8, resultando na Equação 9, onde $\rho_{2}{ }^{\mathrm{L}}$ é a densidade do solvente.

$$
\begin{gathered}
\frac{n_{2}^{L}}{V_{L}}=\frac{\rho_{2}^{L}}{M_{2}} \\
\ln \gamma_{1}^{\infty}=\ln \left(\frac{\rho_{2}^{L} R T}{K_{L g} P_{1}^{\text {sat }} M_{2}}\right)-\frac{P_{1}^{s a t}\left(B_{11}-v_{1}^{0}\right)}{R T}
\end{gathered}
$$

\section{PARTE EXPERIMENTAL}

Os reagentes de grau analítico utilizados foram obtidos de Vetec Química Fina Ltda sendo estes $n$-hexano, $n$-heptano, ciclohexano, furfural e metanol, que tiveram suas purezas comprovadas por análises de cromatografia gasosa, sendo maiores que 99,3\% para todos os solventes. A fibra de PDMS (polidimetilsiloxano) de espessura $30 \mu \mathrm{m}$ foi obtida de Supelco. O cromatógrafo a gás utilizado para análise foi um GC-2010 Shimadzu equipado com detector de ionização em chama (DIC; FID = Flame Ionization Detector), neste foi utilizada uma coluna capilar HP-Innowax (polietileno glicol reticulado; crosslinked PEG) de dimensões $60 \mathrm{~m} \times 0,32 \mathrm{~mm} \times 0,25 \mu \mathrm{m}$; o liner utilizado foi obtido de SGE Analytical Science Pty Ltd e possui diâmetro interno de 0,75 $\mathrm{mm}$ (próprio para SPME), o gás de arraste utilizado foi hélio ultrapuro $(99,999 \%)$. Frascos âmbar de $40 \mathrm{~mL}$ com septo de $\mathrm{PTFE} /$ silicone foram utilizados para o confinamento e estudo das misturas líquidas. 
Todas as análises foram conduzidas com iguais condições cromatográficas, sendo a pressão no injetor mantida constante a 123,6 kPa. Para as análises de injeção de amostra líquida o injetor foi mantido em modo splitless. Foram realizados testes de modo a determinar o tempo necessário para que toda a massa de soluto do injetor fosse transferida para a coluna cromatográfica. O tempo determinado foi 0,5 min, em seguida a válvula split/splitless foi aberta. Para as análises por SPME a válvula split/splitless foi aberta após 4 min para garantir total transferência da massa de soluto e solvente extraída pela fibra. Em todas as análises a válvula de purga foi mantida fechada, de modo a prevenir qualquer perda de amostra injetada. As temperaturas no injetor e detector foram mantidas a $250^{\circ} \mathrm{C}$. A temperatura inicial da coluna foi $75{ }^{\circ} \mathrm{C}$ sendo mantida por 4 min, elevando-se a $150{ }^{\circ} \mathrm{C}$ a uma taxa de aquecimento de $15^{\circ} \mathrm{C} \cdot \mathrm{min}^{-1}$, mantendo-se nesta temperatura por mais $6 \mathrm{~min}$.

As fibras de SPME utilizadas foram condicionadas no injetor do cromatógrafo a gás na temperatura de $250{ }^{\circ} \mathrm{C}$ por $30 \mathrm{~min}$.

As curvas padrão foram construídas pela injeção de $1 \mu \mathrm{L}$ de soluções padrão em metanol dos componentes com concentrações que variaram de 16,5 a $170 \mathrm{ng} / \mu \mathrm{L}$.

Os tempos de extração foram determinados preparando-se amostras gasosas pela injeção de 0,2 a $1 \mu \mathrm{L}$ de soluções metanólicas dos solutos em frascos âmbar de $40 \mathrm{~mL}$ vedados com septo PTFE/silicone. A temperatura no interior dos frascos foi mantida constante por um banho termostático (Lauda, modelo RM 6B) com precisão de $0,1 \mathrm{~K}$. Depois de injetada a amostra e a temperatura ter sido mantida constante, a fibra foi exposta no interior do recipiente por tempo determinado e, em seguida, levada ao injetor do cromatógrafo a gás para a quantificação de material extraído. Os experimentos foram realizados aumentando-se o tempo de exposição até que a massa extraída não variasse mais com o tempo, ou seja, o equilíbrio foi alcançado. O tempo de dessorção adotado foi 4 min com o objetivo de pré-condicionamento da fibra no intervalo dos experimentos e para garantir a total dessorção de eventuais contaminantes.

Para a determinação dos coeficientes de partição entre a fase polimérica de PDMS da fibra de SPME e o soluto na fase gasosa a $298,15 \mathrm{~K}\left(25^{\circ} \mathrm{C}\right)$ foram preparadas amostras gasosas dos solutos, de forma análoga às amostras preparadas para determinação dos tempos de extração. A fibra foi exposta na amostra durante o tempo de extração previamente determinado e, então, levado ao injetor do cromatógrafo a gás para quantificação do material extraído.

A determinação dos coeficientes de atividade em diluição infinita foi realizada pelo preparo de soluções de hidrocarbonetos em furfural, em frações molares que variaram de 0,00015 a 0,00025 . Para cada uma das soluções tomou-se uma alíquota de $10 \mathrm{~mL}$. Estas foram postas separadamente em frascos âmbar de $40 \mathrm{~mL}$ que, em seguida, foram fechados com tampa e septo de PTFE/silicone. Os frascos foram submetidos à agitação por tempos de 30, 45 e 60 min separadamente, de modo a se determinar o tempo de equilíbrio. Após a agitação a temperatura do sistema foi mantida a $25^{\circ} \mathrm{C}$ por 45 min utilizando um banho termostático. Em seguida, a fibra foi exposta no headspace da amostra e depois exposta no injetor do cromatógrafo a gás para a quantificação da massa extraída. O coeficiente de partição entre a fase líquida e a fase gasosa foi determinado pela Equação 6 e o coeficiente de atividade em diluição infinita foi determinado pela Equação 9 .

As pressões de vapor dos solutos a 298,15 K foram calculadas pela Equação de Wagner (Equação 10). As constantes para o soluto podem ser encontradas na literatura. ${ }^{13}$

$\ln \left(\frac{P^{v a p}}{P c}\right)=\frac{A(1-T r)+B(1-T r)^{1,5}+C(1-T r)^{3}+D(1-T r)^{6}}{T r}$ onde $\operatorname{Tr}$ é a temperatura reduzida do soluto e Pc a pressão crítica do soluto.

O segundo coeficiente de Virial foi calculado para cada soluto pelo método de Tsonopoulos ${ }^{14,15}$ que propôs:

$$
z=1+\left(\frac{B_{i i} P_{c}}{R T_{c}}\right) \frac{P_{r}}{T_{r}}=z^{(0)}+w z^{(1)}
$$

onde $z^{(0)}$ é o fator de compressibilidade para fluidos simples e $z^{(1)}$ descreve os desvios significativos à forma esférica e que necessita de correções. $\mathrm{O}$ fator acêntrico $w$ mede o desvio em relação à esfericidade. Como $B_{i i} P_{c} / R T_{c}$ é adimensional, tem-se:

$$
\frac{B_{i i} P_{c}}{R T_{c}}=F_{w}\left(T_{r}\right)=b^{(0)}\left(T_{r}\right)+w b^{(1)}\left(T_{r}\right)
$$

onde $F_{w}$ é uma função generalizada que depende do fator acêntrico e tem os valores de $b^{(0)} \mathrm{e} b^{(1)}$ determinados pelas relações empíricas. ${ }^{14}$

$$
\begin{array}{r}
b^{(0)}=0,1445-\frac{0,330}{T_{r}}-\frac{0,1385}{T_{r}^{2}}-\frac{0,0121}{T_{r}^{3}}-\frac{0,000607}{T_{r}^{8}} \\
b^{(1)}=0,0637+\frac{0,331}{T_{r}^{2}}-\frac{0,423}{T_{r}^{3}}-\frac{0,008}{T_{r}^{8}}
\end{array}
$$

Foi determinada a razão de solvente absorvida pelo revestimento da fibra pela Equação 15, de modo a controlar um possível inchamento deste revestimento.

$$
\%(\text { inchamento })=\frac{m_{\text {solv }}}{\rho_{f} V_{f}} \cdot 100
$$

\section{RESULTADOS E DISCUSSÃO}

As equações da reta obtidas pela curva padrão para os componentes analisados e os coeficientes de determinação $\left(\mathrm{R}^{2}\right)$ são apresentados na Tabela 1 .

Tabela 1. Dados das curvas padrão

\begin{tabular}{lccc}
\hline & \multicolumn{3}{c}{ Equação da reta $(\mathrm{y}=\mathrm{ax}+\mathrm{b})$} \\
Componente & $\mathrm{a}$ & $\mathrm{b}$ & $\mathrm{R}^{2}$ \\
\hline$n$-Hexano & 18556 & -27832 & 0,9998 \\
$n$-Heptano & 17482 & -54002 & 0,9995 \\
Ciclo-hexano & 17566 & -75905 & 0,9977 \\
Furfural & 7662 & -343971 & 0,9958 \\
\hline
\end{tabular}

Os valores de $\mathrm{B}_{11}, \mathrm{P}_{1}^{\text {sat }} \mathrm{e}_{1}{ }^{\circ}$ são apresentados na Tabela 2. Os valores de $\mathrm{v}_{1}{ }^{\circ}$ foram obtidos da literatura. ${ }^{1}$

Tabela 2. Valores das propriedades dos solutos a $298,15 \mathrm{~K}$

\begin{tabular}{lccc}
\hline Soluto & $\mathrm{B}_{11}\left(\mathrm{~cm}^{3} / \mathrm{mol}\right)$ & $\mathrm{v}_{1}{ }^{\circ}\left(\mathrm{cm}^{3} / \mathrm{mol}\right)$ & $\mathrm{P}_{1}$ sat $(\mathrm{Pa})$ \\
\hline$n$-Hexano & -1973 & 131,6 & 20152 \\
$n$-Heptano & -3037 & 147,5 & 6073 \\
Ciclo-hexano & -1884 & 108,7 & 13039 \\
\hline
\end{tabular}

Os tempos de extração determinados para cada soluto são apresentados na Tabela 3. Após a dessorção a fibra foi exposta no injetor do cromatógrafo para se verificar a possibilidade de material não dessorvido, o que mostrou uma não variação da linha de base, indicando a ausência de material não dessorvido. 
Tabela 3. Tempos de extração dos solutos

\begin{tabular}{lccc}
\hline Soluto & Fibra & $\begin{array}{c}\text { Massa injetada } \\
(\mu \mathrm{g})\end{array}$ & $\begin{array}{c}\text { Tempo de extração } \\
(\mathrm{min})\end{array}$ \\
\hline$n$-Hexano & PDMS 30 $\mu \mathrm{m}$ & 66,0 & 2,00 \\
$n$-Heptano & PDMS 30 $\mu \mathrm{m}$ & 27,2 & 2,00 \\
Ciclo-hexano & PDMS 30 $\mu \mathrm{m}$ & 39,0 & 2,00 \\
\hline
\end{tabular}

Os valores dos coeficientes de partição entre o PDMS e soluto na fase gasosa são confrontados com os resultados da literatura e estes são apresentados na Tabela 4.

Tabela 4. Coeficientes de partição soluto (gás) - PDMS a 298,15 K comparados com os valores da literatura

\begin{tabular}{lcccc}
\hline Soluto & \multicolumn{4}{c}{$\mathrm{K}_{298,15}$} \\
& $\begin{array}{c}\text { SPME } \\
\text { (este trabalho) }\end{array}$ & SPME $^{16}$ & LTPRI $^{8}$ & GLC $^{17}$ \\
\hline$n$-Hexano & 213 & 170 & 215 & 166 \\
$n-$ Heptano & 609 & - & 565 & 451 \\
Ciclo-hexano & 408 & - & - & - \\
\hline
\end{tabular}

Pode-se notar que alguns valores de coeficientes de partição se distanciam dos valores encontrados na literatura, porém estes desvios não são expressivos. Ainda é possível afirmar que mesmo apresentando desvios, os valores dos coeficientes de partição podem variar de acordo com a técnica utilizada para sua determinação, como pode ser visto na literatura. ${ }^{8,16,17}$

A Figura 2 mostra um típico cromatograma da extração por SPME na fase vapor (headspace) em um recipiente contendo uma mistura líquida de $n$-hexano e furfural.

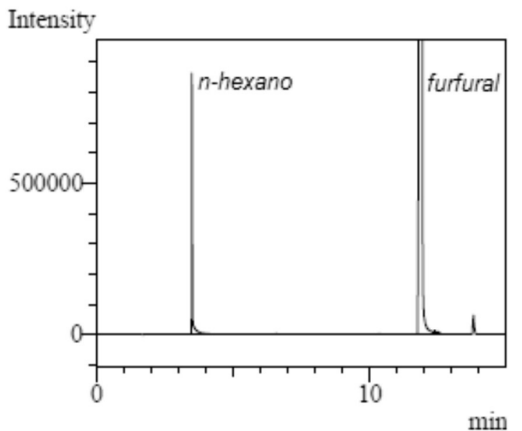

Figura 2. Cromatograma do n-hexano e furfural extraídos por SPME

Os coeficientes de atividade na diluição infinita determinados pela técnica de SPME são mostrados na Tabela 5 e confrontados com os dados da literatura. Os desvios entre os métodos foram calculados pela Equação 16.

$$
\operatorname{Desvio}(\%)=\frac{\left|\gamma_{\text {Lit. }}^{\infty}-\gamma_{S P M E}^{\infty}\right|}{\gamma_{\text {Lit. }}^{\infty}} \cdot 100
$$

onde $\gamma_{\text {Lit. }}^{\infty}$ é o coeficiente de atividade encontrado na literatura e $\gamma_{\text {SPME }}^{\infty}$ é o coeficiente determinado pela técnica de SPME.

\section{CONCLUSÃO}

Tendo por base os valores encontrados para os coeficientes de atividade em diluição infinita, é possível afirmar que a SPME é uma
Tabela 5. Coeficientes de atividade na diluição infinita comparados com os valores da literatura

\begin{tabular}{lcccc}
\hline Soluto & & \multicolumn{3}{c}{$\gamma_{i}^{\infty}$} \\
& SPME & Literatura & Desvio (\%) & Referência \\
\hline$n$-Hexano & $25,2 \pm 0,3$ & $30,0 \pm 1,8$ & 16,00 & GLC $^{18}$ \\
& & $26,0 \pm 0,5$ & 3,10 & Gas Stripping $^{18}$ \\
& & $28,5 \pm 1,7$ & 11,58 & $\begin{array}{c}\text { GLC/Gas } \\
\text { Stripping }\end{array}$ \\
& & & & GLC $^{19}$ \\
Ciclohexano & \multirow{2}{*}{$14 \pm 0,6$} & $17,5 \pm 0,4$ & 20,0 & GLC $^{18}$ \\
& & $14,0 \pm 0,3$ & 0,00 & Gas stripping $^{18}$ \\
& & $13,6 \pm 0,5$ & 2,94 & GLC $^{1}$ \\
$n-$ Heptano & $31,9 \pm 0,7$ & 33,4 & 4,49 & GLC $^{1}$ \\
\hline
\end{tabular}

técnica válida para a determinação destes coeficientes, pois mesmo com as aproximações feitas os resultados foram satisfatórios, obtendo-se um desvio médio dos valores da literatura de 7\%. Apenas dois resultados diferem da literatura com desvios de $16 \%$ para o $n$-hexano e $20 \%$ para o ciclo-hexano. Contudo, estes coeficientes podem ser considerados válidos, visto que entre os próprios resultados da literatura há discrepâncias maiores. Além disso, a SPME é uma técnica mais simples de ser implementada e utilizada do que técnicas como o GLC e o gas-stripping.

Algumas limitações foram observadas na utilização da técnica de SPME para a determinação destes coeficientes. Uma destas é a possibilidade de inchamento do revestimento polimérico da fibra de SPME, que quando retraída pode ocasionar a raspagem deste material ou mesmo a quebra da fibra. Logo, não é recomendável utilizar solventes cuja afinidade pela fibra seja demasiadamente alta. Tal observação mostra que na determinação do coeficiente de atividade em solvente polar faz-se necessária uma fibra apolar, o que limita a utilização da técnica a sistemas de polaridades opostas, no qual a fibra tem a mesma característica de polaridade do soluto. Mesmo com a limitação observada, a técnica de SPME ainda pode ser vista de modo vantajoso se comparada à técnica de GLC, que é utilizada apenas para solventes não voláteis. A evolução da tecnologia de fabricação das fibras de SPME com materiais de polaridades e espessuras distintas permitirá que se determine o coeficiente de atividade em qualquer sistema de polaridades opostas, mesmo que ambos sejam voláteis. Isto vai possibilitar a obtenção dos extremos de diluição para cada componente e a imediata determinação de parâmetros de modelos matemáticos que descrevam o equilíbrio de fases como, por exemplo, o modelo de Wilson, cujas constantes $\left(\Lambda_{\mathrm{ij}} \mathrm{e} \Lambda_{\mathrm{jij}}\right)$ são independentes da composição. Com estas constantes é possível o cálculo de coeficientes de atividade em quaisquer outras composições. Por exemplo, usando as equações de Wilson na diluição infinita, temos $\ln \gamma_{i}^{\infty}=-\ln \Lambda_{\mathrm{ij}}+1-\Lambda_{\mathrm{ji}}$ $\mathrm{e} \ln \gamma_{j}^{\infty}=-\ln \Lambda_{\mathrm{ji}}+1-\Lambda_{\mathrm{ij}}$. Dispondo de valores experimentais de $\gamma_{i}^{\infty} \mathrm{e} \gamma_{j}^{\infty}$, determinam-se imediatamente as constantes de Wilson, características das interações entre os componentes $i$ e $j$ em solução.

Outro fator importante a ser analisado é a fração mássica de solvente absorvido pelo revestimento. Uma fração de solvente que represente mais de $1 \%$ da massa do revestimento pode alterar os coeficientes de partição reais entre um soluto e este mesmo revestimento; isto se dá por interações entre os componentes adsorvidos/ absorvidos. ${ }^{20}$ Uma forma de minimizar a quantidade de solvente extraída seria utilizar fibras com espessuras menores, visto que quanto menor a espessura do revestimento polimérico menor será o tempo de equilíbrio entre soluto e revestimento.

Em todas as medições foram observadas frações de solvente que representavam entre 4 e $4,5 \%$ da massa do revestimento, porém foi 
observado que este solvente não interferiu nos coeficientes de partição, visto os baixos desvios dos valores de coeficientes de atividade da literatura.

\section{REFERÊNCIAS}

1. Letcher T. M.; Kozlowska, M. K.; Zelasna, U. D.; J. Chem. Thermodyn. 2004, 36, 37.

2. Mariano, J. B.; Dissertação de Mestrado, Universidade Federal do Rio de Janeiro, Brasil, 2001.

3. Cruickshank, A. J. B.; Gainey, B. W.; Hicks, C. P.; Letcher, T. M.; Moody, R. W.; Young, C. L.; Trans. Faraday Soc. 1969, 65, 1014.

4. Everett, D. H.; Trans. Faraday Soc. 1965, 61, 1637.

5. Bao, J. B.; Han, S. J.; Fluid Phase Equilib. 1995, 112, 307.

6. Haimi, P.; Uusi-Kyyny, P.; Pokki, J. P.; Aittamaa, J.; Keskinen, K. I.; Fluid Phase Equilib. 2006, 243, 126.

7. Berezkin, V. G.; Alishoyev, V. R.; Nemirovskaya, I. B.; Gas chromatography of polymers, Elsevier: Amsterdan, 1977.

8. Martos, P. A.; Saraullo, A.; Pawliszyn, J.; Anal. Chem. 1997, 69, 402.
9. Zhang, Z.; Pawliszyn, J.; J. Phys. Chem. 1996, 100, 17648.

10. Fonseca, D. B.; Coelho, G. L. V.; Quim. Nova 2007, 30, 1606.

11. Valente, A. L. P.; Augusto, F.; Quim. Nova 2000, 23, 523.

12. Gaurav; Kaur,V.; Kumar, A.; Malik, A. K.; Rai, P. K.; J. Hazard. Mater. 2007, 147, 691.

13. Reid, R. C.; Prausnitz, J. M.; Poling, B. E.; The Properties of Gases and Liquids, $4^{\text {th }}$ ed., McGraw-Hill: New York, 1988.

14. Tsonopoulos, C.; AIChE J. 1974, 20, 263.

15. Gmehling, G.; Kolbe, B.; Thermodynamik; George Thieme Verlag: Sttutgart, 1988, cap. 2.

16. Martos, P. A.; Pawliszyn, J.; Anal. Chem. 1997, 69, 206.

17. Kloskowski, A.; Chrzanowski, W.; Pilarczyk, M.; Namiesnik, J.; J. Chem. Thermodyn. 2005, 37, 21.

18. Santacesaria, E.; Berlendis, D.; Carrà, S.; Fluid Phase Equilib. 1979, 3 , 167.

19. Deal, C. H.; Derr, E. L.; Ind. Eng. Chem. Process Des. Dev. 1964, 3, 394.

20. Pawliszyn, J.; Solid Phase Microextraction: Theory and Practice, WileyVHC: New York, 1997. 